\title{
Virulence factors and antibiotic resistance of avian pathogenic Escherichia coli in eastern China
}

\author{
Xiaojing Xu${ }^{1}$, Qing Sun ${ }^{2,3}$, Lixiang Zhao ${ }^{1}$ \\ ${ }^{1}$ College of Basic Medicine and Biological Sciences, Medical Department, \\ Soochow University, 215123, Suzhou, People's Republic of China \\ ${ }^{2}$ Laboratory of Animal Infectious Diseases, College of Veterinary Medicine, \\ Yangzhou University, Yangzhou, 225009, People's Republic of China \\ ${ }^{3}$ Virus Research Unit, Department of Microbiology and Immunology, \\ School of Medicine, University of Otago, 9016, Dunedin, New Zealand \\ zhaolixiang@suda.edu.cn
}

Received: February 15, $2019 \quad$ Accepted: August 7, 2019

\begin{abstract}
Introduction: Avian pathogenic Escherichia coli (APEC) causes serious colibacillosis and significant economic losses. Data on profiles of virulence factors and antibiotic resistances among APEC strains are crucial to the control of infection. In this study, strains were isolated from eastern China, and the prevalence of virulence factors and distribution of antibiotic resistance were determined. Material and Methods: APEC strains were isolated and characterised by PCR for O serogroups, virulence factor genes, antibiotic resistance, and phylogenetic groups. Results: O78 was the most prevalent serogroup and type A was the most frequent phylogenetic group. The $\mathrm{fimH}, \mathrm{feoB}$, and iron genes were the most prevalent among the isolates. All isolates were multiresistant, and all strains were resistant to ampicillin and tetracycline, which are widely used in the poultry industry in China. Conclusion: This study provided important data on the presence of virulence genes and antibiotic resistance profiles of APEC from poultry farms in eastern China.
\end{abstract}

Keywords: chicken, Escherichia coli, phylogenetic group, virulence factors, antibiotic resistance.

\section{Introduction}

Escherichia coli is considered a normal part of the intestinal microflora in humans and birds. Certain avian pathogenic E. coli strains (APEC) are accepted to be the main causes of avian colibacillosis, which manifests as pericarditis, perihepatitis, peritonitis, airsacculitis, and septicaemia, and causes significant economic losses worldwide every year $(1,8)$. The pathogenic ability of APEC is mediated by a broad range of virulence factors, such as colonisation factors (facilitating attachment of APEC to extraintestinal tracts), adhesins (also facilitating attachment of APEC to extraintestinal tracts and assisting penetration of bacteria into the tissues), toxins (protecting APEC from lysosomes), siderophores (chelating iron), and protectins (inhibiting the classical pathway of complement activity), which help the bacterial infection to become established and augment the bacterium's resistance to the host's immune defences. Epidemic data show that human extraintestinal pathogenic E. coli (ExPEC) strains and APEC often carry similar virulence genes, suggesting the zoonotic importance of APEC strains.

Antibacterial drugs have been widely used in the poultry industry to control APEC infection (4). In many countries, including China, antibacterial drugs have been used as therapeutic methods and for prevention of infection and growth promotion (5). However, excessive use of antimicrobials in food-producing animals has led to several adverse effects on animals, humans, and the environment. Meanwhile continuous administration of antibacterial drugs leads to the emergence of multidrug-resistant strains (MDR) of E. coli (6). These strains can be transmitted to people through the food chain resulting in a serious risk to human health. Drug resistance has consequently become a focus of interest for both the poultry industry and public healthcare in China.

Data on profiles of virulence factors and antibiotic resistances among APEC strains are crucial to infection control in China. In this study, we isolated strains from eastern China and determined the prevalence of 
virulence factors, distribution of antibiotic resistance, and phylogenetic background of these isolates.

\section{Material and Methods}

Bacterial strains. Samples of the liver, lungs, air sacs, and spleen from infected chickens with typical lesions of $E$. coli infections were collected from farms in the Jiangsu and Shandong provinces from 2005 to 2008. Samples were cultured on MacConkey agar overnight at $37^{\circ} \mathrm{C}$, and suspect bacterial colonies were further isolated on LB agar. The isolates were kept at $-70^{\circ} \mathrm{C}$ with the addition of $20 \%(\mathrm{v} / \mathrm{v})$ glycerol. For each sample, only one colony was isolated and used for subsequent examination. The serotyping of each strain was carried out according to the standard methods of the Chinese Institute of Veterinary Drug Control. Briefly, 21 sets of polyvalent antisera against $174 \mathrm{O}$ serogroups (Tianjin Biochip Corporation, Tianjin, China) were used to determine the $\mathrm{O}$ serogroups of each strain using agglutination tests according to the manufacturer's instructions.

Phylogenetic type and gene virulence detection. Bacterial DNA were extracted using Rapid DNA extraction kits according to the manufacturer's protocol (Tiangen Biotech, Beijing, China). The phylogenetic types were determined by a PCR targeting three genes (ChuA, YjaA, and TSPE4.C2) as previously described (11). Virulence genes totalling 33 were determined by a PCR targeting iucC and six sets of multiplex PCRs targeting different sets of the following genes: 1) papA, fimH, kpsMT III, papEF, ireA, and ibeA; 2) cnf-1, fyuA, iroN, bmaE, sfa, iutA, and papG allele III; 3) $h l y D, r f c, o m p T, p a p G$ allele I , kpsMT II, and papC; 4) gafD, $c d t B$, traT, and pap $G$ allele II; 5) pap $G$ allele I, iha, afa, iss, sfaS, and kpsMT (K1); and 6) sitA, feoB, and irp-2 (11).

Antimicrobial resistance testing. A total of nine antimicrobial drugs (ampicillin (AMP $10 \mu \mathrm{g}$ ), cefotaxime (CTX $30 \mu \mathrm{g})$, chloramphenicol (30 $\mu \mathrm{g})$, gentamicin $(10 \mu \mathrm{g})$, kanamycin $(30 \mu \mathrm{g})$, nalidixic acid
(30 $\mu \mathrm{g})$, streptomycin $(10 \mu \mathrm{g})$, sulphamethoxazoletrimethoprim (STX 1.25/23.75 $\mu \mathrm{g}$ ), and tetracycline (TET $30 \mu \mathrm{g}$ )) were used for antimicrobial susceptibility testing by the Kirby-Bauer method according to the National Committee for Clinical Laboratory Standards interpretive criteria.

\section{Results}

Serotyping of the isolates. In total 106 APEC strains were isolated and $77(72.64 \%)$ strains were $\mathrm{O}$-antigen typable. Most of the O-antigen typable strains belonged to $\mathrm{O} 78$ (19 isolates), O2 (6 isolates), O18 (6 isolates), $\mathrm{O} 45$ (5 isolates), $\mathrm{O} 1$ (5 isolates), and $\mathrm{O} 4$ (3 isolates).

Phylogenetic typing. Phylogenetic types were determined using PCR, and phylotype A proved to be the most prevalent phylogenetic group, to which $44.34 \%$ (47 out of 106) affiliated. In the remaining 59 isolates, 32 belonged to phylogenetic group B2, 15 to B1, and 12 to $\mathrm{D}$ (Table 1). The correlation of the O-serotype with the phylogenetic group was also examined (Table 2).

Genotyping of virulence genes. The prevalence of 33 virulence genes in each APEC strain was screened by PCR (Table 3). The results showed that fim H, feoB, and iron were the most prevalent genes among all isolates, exceeding $90 \%$ in each case. More than $70 \%$ of isolates also carried the ireA, irp-2, iutA, ompT, iss, or traT genes. Four virulence genes (iha, $r f c, c n f-1$, and $h l y D)$ were not detected in this study.

Antimicrobial resistance. Antimicrobial resistance test results showed that the isolates were commonly resistant to the tested antibiotics (Table 4). All isolates exhibited resistance to ampicillin and tetracycline. The frequencies of resistance to other antimicrobials were $89.62 \%$ to nalidixic acid, $83.96 \%$ to chloramphenicol, $80.19 \%$ to kanamycin, $64.15 \%$ to streptomycin, $58.49 \%$ to sulphamethoxazole-trimethoprim, $53.77 \%$ to cefotaxime, and $50.00 \%$ to gentamicin. All of the isolates were multi-drug-resistant (MDR) strains.

Table 1. Phylogenetic groups of 106 APEC strains

\begin{tabular}{lll}
\hline Phylogenetic group & Number & Percentage \\
\hline A & 47 & 43.34 \\
B1 & 15 & 14.15 \\
B2 & 32 & 30.19 \\
D & 12 & 11.32 \\
\hline
\end{tabular}

Table 2. Correlation of the O-serotype with the phylogenetic group

\begin{tabular}{|c|c|c|c|c|c|}
\hline \multirow{2}{*}{ O-serotype } & \multicolumn{4}{|c|}{ Phylogenetic group } & \multirow[b]{2}{*}{ Total } \\
\hline & $\mathrm{A}$ & B1 & B2 & $\mathrm{D}$ & \\
\hline $\mathrm{O} 1$ & 2 & 2 & 1 & 0 & 5 \\
\hline $\mathrm{O} 2$ & 3 & 2 & 0 & 1 & 6 \\
\hline $\mathrm{O} 4$ & 2 & 0 & 1 & 0 & 3 \\
\hline $\mathrm{O} 18$ & 4 & 2 & 0 & 0 & 6 \\
\hline $\mathrm{O} 45$ & 2 & 1 & 1 & 1 & 5 \\
\hline $\mathrm{O} 78$ & 8 & 5 & 3 & 3 & 19 \\
\hline
\end{tabular}


Table 3. Prevalences of virulence factors in 106 APEC strains

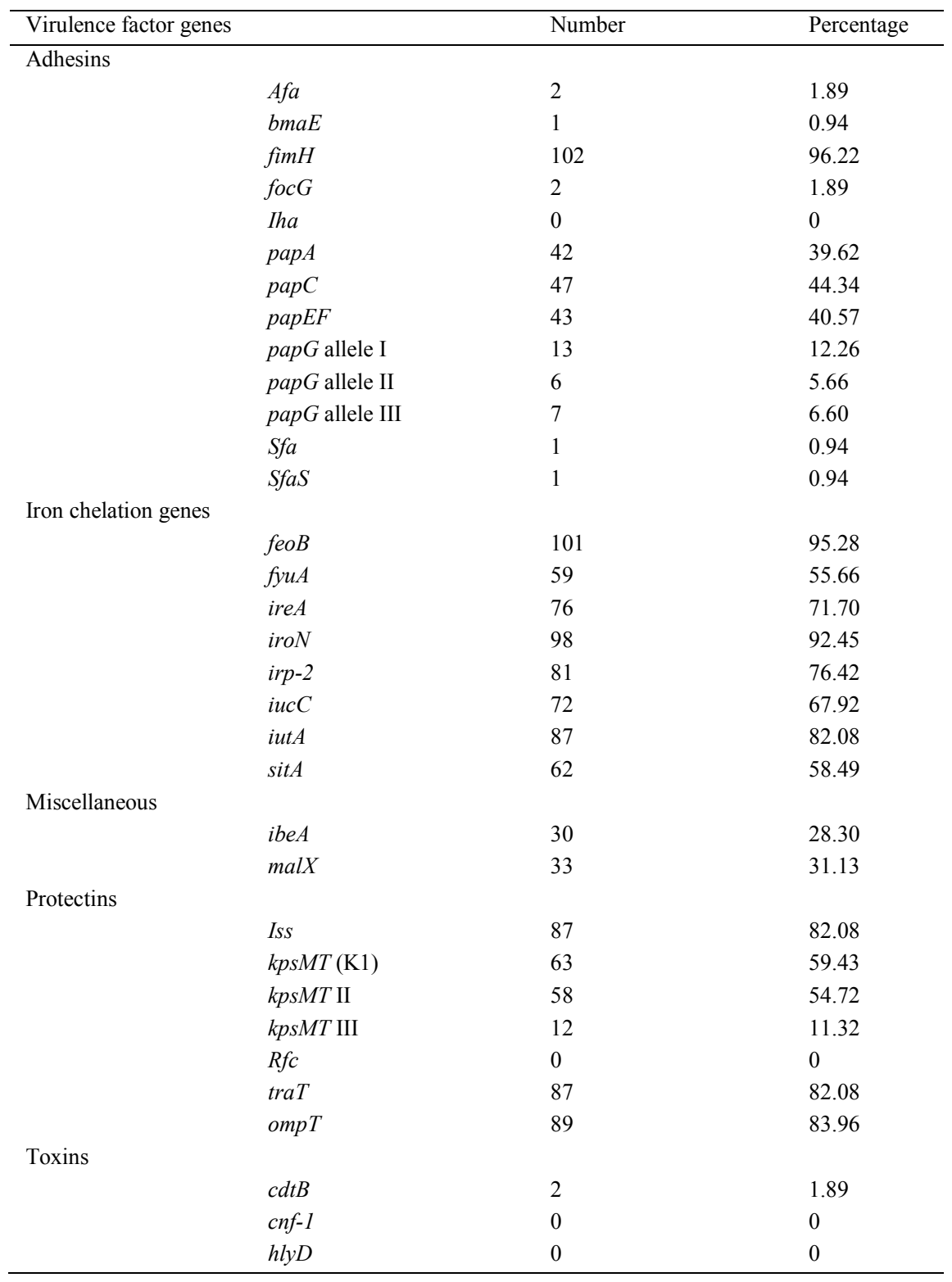

Table 4. Antibiotic resistance of 106 APEC strains

\begin{tabular}{lll}
\hline Antibiotics & Number of resistant strains & Percentage of resistant strains \\
\hline Ampicillin & 106 & 100 \\
Cefotaxime & 57 & 53.77 \\
Chloramphenicol & 89 & 83.96 \\
Gentamicin & 53 & 50.00 \\
Kanamycin & 85 & 80.19 \\
Streptomycin & 68 & 64.15 \\
Sulphamethoxazole-trimethoprim & 62 & 58.49 \\
Nalidixic acid & 95 & 89.62 \\
Tetracycline & 106 & 100 \\
\hline
\end{tabular}

\section{Discussion}

The poultry industry is developing rapidly in many regions of China. Colibacillosis is one of the major diseases blighting the poultry industry and antibiotics are widely used in its control. However, the emergence of multi-drug-resistant APEC strains has often led to the failure of antibiotics to control APEC infections. In many regions of China, data on virulence genes and antibiotic resistance of APEC are often unavailable. In the present study, APEC strains were isolated from chickens affected with colibacillosis, and virulence factor genes and antibiotic resistance were determined.

APEC isolates often carried a broad range of virulence genes. O-antigens, one of the welldocumented virulence factors of $E$. coli, can protect bacteria from clearance by the neutrophils and macrophages of the host (2). A specific O-antigen (such as $\mathrm{O} 1, \mathrm{O} 2$, and $\mathrm{O} 78$ ) is often associated with the virulence of APEC strains (3), and APEC isolates with 
$\mathrm{O}$-antigens are considered virulent. Here, we found that $\mathrm{O} 78$ and $\mathrm{O} 2$ are the dominant strains among APEC isolates from eastern China, which is consistent with other research from Egypt (10), suggesting wide distribution of these two O-antigens in virulent APEC.

Adhesin is the most important gene which helps the bacteria colonise host cells to build infection. Most APEC isolates from the current study carried one of the most important adhesin-encoding genes, fimH. The pap gene cluster, encoding a pilus rod, was also present in high prevalence. The $s f a$ gene, encoding $\mathrm{S}$ fimbriae, occurred in very low prevalence. $S$ fimbriae, one of the mannose-resistant adhesins, often occurred in other ExPEC strains, namely strains of uropathogenic E. coli, and the very low prevalence of $s f a$ in APEC suggested the important role of this adhesin in urinary infections rather than in avian infection by ExPEC (7).

The polysialic acid capsule-encoding gene $($ kpsMT) is one of the important virulence factors which shield $E$. coli from lysis by lysosomes, and over $50 \%$ of APEC isolates carry this gene. OmpT promotes formation of bacterial communities during APEC infection, and more than $80 \%$ of APEC isolates possess it, suggesting the essential role of this gene in APEC infection.

Excessive use of antimicrobials in chicken has led to several adverse effects on animals, humans, and the environment. Our data demonstrated that the antibiotic resistance of APEC isolated from eastern China is a serious concern. Out of nine antibiotics tested, none showed more than $50 \%$ effectiveness against the APEC strains, and as the least resisted antibiotic, gentamicin was inefficacious with $50 \%$ of APEC strains. All APEC strains were resistant to ampicillin and tetracycline. It could result from the wide use of these antibiotics in the poultry industry in eastern China. About $90 \%$ of strains in this study were resistant to nalidixic acid, which was a higher percentage than strains isolated from Belgium and North Georgia $(9,12)$. The reason may be the relatively high utilisation of nalidixic acid in eastern China.

In summary, the APEC isolates in the present study carried a set of virulence factors, and these isolates also exhibited high rates of resistance to commonly used antibiotics, which could cause a serious public health problem.

Conflict of Interests Statement: The authors declare that there is no conflict of interests regarding the publication of this article.

Financial Disclosure Statement: This work was supported by the National Natural Science Foundation of China (31870918 and 31500746), the Natural Science Fund of Jiangsu province (BK20170334), the Open Project Program of Jiangsu Key Laboratory of Zoonosis (number R1601), project funding from Suzhou City (SNG2017047 and SYS201677), the Training Programs of Innovation and Entrepreneurship for Undergraduates of Soochow University (201510285105X), and a project funded by the Priority Academic Program Development of Jiangsu higher education institutions.

Animal Rights Statement: The authors declare that the experiments on animals were conducted in accordance with local Ethical Committee laws and regulations as regards care and use of laboratory animals.

\section{References}

1. Chaudhari A.A., Kariyawasam S.: An experimental infection model for Escherichia coli egg peritonitis in layer chickens. Avian Dis 2014, 58, 25-33.

2. Crémet L., Broquet A., Jacqueline C., Chaillou C., Asehnoune K., Corvec S., Caroff N.: Innate immune evasion of Escherichia coli clinical strains from orthopedic implant infections. Eur J Clin Microbiol Infect Dis 2016, 35, 993-999.

3. Ewers C., Janssen T., Kiessling S., Philipp H.C., Wieler L.H.: Molecular epidemiology of avian pathogenic Escherichia coli (APEC) isolated from colisepticemia in poultry. Vet Microbiol 2004, 104, 91-101

4. Ghunaim H., Abu-Madi M.A., Kariyawasam S.: Advances in vaccination against pathogenic Escherichia coli respiratory disease: potentials and limitations. Vet Microbiol 2014, 172, 13-22.

5. Krishnasamy V., Otte J., Silbergeld E.: Antimicrobial use in Chinese swine and broiler poultry production. Antimicrob Resist Infect Control 2015, 4, 17

6. Li Y., Chen L., Wu X., Huo S.: Molecular characterization of multidrug-resistant avian pathogenic Escherichia coli isolated from septicemic broilers. Poult Sci 2015, 94, 601-611.

7. Obaid J.M., Mansour S.R., Elshahedy M.S., Rabie T.E., Azab A.M.: Uropathogenic Escherichia coli isolates with different virulence genes content exhibit similar pathologic influence on Vero cells. Pol J Microbiol 2014, 63, 43-49.

8. Oliveira A., Sereno R., Azeredo J.: In vivo efficiency evaluation of a phage cocktail in controlling severe colibacillosis in confined conditions and experimental poultry houses. Vet Microbiol 2010, 146, 303-308.

9. Oosterik L.H., Peeters L., Mutuku I., Goddeeris B.M., Butaye P.: Susceptibility of avian pathogenic Escherichia coli from laying hens in Belgium to antibiotics and disinfectants and integron prevalence. Avian Dis 2014, 58, 271-278.

10. Ramadan H., Awad A., Ateya A.: Detection of phenotypes, virulence genes, and phylotypes of avian pathogenic and human diarrheagenic Escherichia coli in Egypt. J Infect Dev Ctries 2016, $10,584-591$

11. Zhao L., Gao S., Huan H., Xu X., Zhu X., Yang W., Gao Q., Liu X.: Comparison of virulence factors and expression of specific genes between uropathogenic Escherichia coli and avian pathogenic $E$. coli in a murine urinary tract infection model and a chicken challenge model. Microbiology 2009, 155, 1634-1644.

12. Zhao S., Maurer J.J., Hubert S., De Villena J.F., McDermott P.F., Meng J., Ayers S., English L., White D.G.: Antimicrobial susceptibility and molecular characterization of avian pathogenic Escherichia coli isolates. Vet Microbiol 2005, 107, 215-224. 